\title{
Library of high-resolution UES echelle spectra of F, G, K and $M$ field dwarf $\operatorname{stars}^{\star, \star \star, \star \star \star}$
}

\author{
D. Montes ${ }^{1,2}$ and E.L. Martín ${ }^{3,4}$ \\ 1 Departamento de Astrofísica, Facultad de Físicas, Universidad Complutense de Madrid, E-28040 Madrid, Spain \\ 2 Pennsylvania State University, Department of Astronomy \& Astrophisics, 525 Davey Lab., University Park, PA 16802, U.S.A. \\ 3 Instituto de Astrofísica de Canarias, E-38200 La Laguna, Tenerife, Spain \\ ${ }^{4}$ University of California at Berkeley, Department of Astronomy, Berkeley, CA 94720, U.S.A.
}

Received June 20; accepted August 12, 1997

\begin{abstract}
We present a library of Utrecht echelle spectrograph (UES) observations of a sample of F, G, K and M field dwarf stars covering the spectral range from $4800 \AA$ to $10600 \AA$ with a resolution of 55000 . These spectra include some of the spectral lines most widely used as optical and near-infrared indicators of chromospheric activity such as $\mathrm{H} \beta, \mathrm{Mg}$ I b triplet, $\mathrm{Na}$ I $\mathrm{D}_{1}, \mathrm{D}_{2}$, $\mathrm{He}$ I $\mathrm{D}_{3}, \mathrm{H} \alpha$, and Ca II IRT lines, as well as a large number of photospheric lines which can also be affected by chromospheric activity. The spectra have been compiled with the aim of providing a set of standards observed at high-resolution to be used in the application of the spectral subtraction technique to obtain the active-chromosphere contribution to these lines in chromospherically active single and binary stars. This library can also be used for spectral classification purposes. A digital version with all the spectra is available via ftp and the World Wide Web (WWW) in both ASCII and FITS formats.
\end{abstract}

Key words: Atlases - stars: activity - stars: chromospheres - stars: late-type - stars: fundamental parameters - stars: general

Send offprint requests to: D. Montes (dmg@astrax.fis.ucm.es) * Based on observations made with the William Herschel Telescope operated on the island of La Palma by the Isaac Newton Group at the Spanish Observatorio del Roque de Los Muchachos of the Instituto de Astrofísica de Canarias.

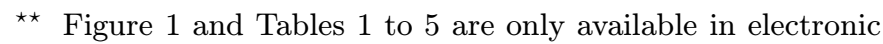
form, and Table 6 is also available in electronic form.

${ }^{\star \star \star}$ The spectra of the stars listed in Table 6 are also available in electronic form at the CDS via anonymous ftp to cdsarc.u-strasbg.fr (130.79.128.5) or via http://cdsweb.ustrasbg.fr/Abstract.html

\section{Introduction}

Spectral libraries of late-type stars are a very powerful tool for the study of the chromospheric activity by application of the spectral subtraction technique (see Montes et al. 1995a,b,c; and references therein). Furthermore, these libraries are also very useful in many areas of astrophysics such as the stellar spectral classification, modelling stellar atmospheres, stellar abundances, calibration of temperatures, spectral synthesis applied to composite systems, and spectral synthesis of the stellar population of galaxies.

While for many of this purposes, obtaining as large a spectral range as possible was the main priority, for the chromospheric activity studies, which are centered in specific spectral features, it is much more important to increase the spectral resolution. However, previously published stellar libraries are of poor spectral resolution (between 45 and $1.25 \AA$ ) and the only attempt to improve the spectral resolution is our library of high and midresolution spectra in the $\mathrm{Ca}$ II $\mathrm{H} \& \mathrm{~K}, \mathrm{H} \alpha, \mathrm{H} \beta, \mathrm{Na}$ I $\mathrm{D}_{1}$, $\mathrm{D}_{2}$, and $\mathrm{He}$ I $\mathrm{D}_{3}$ line regions of $\mathrm{F}, \mathrm{G}, \mathrm{K}$ and $\mathrm{M}$ field stars (Montes et al. 1997a, hereafter Paper I) with resolutions that range between 3 and $0.2 \AA$.

However, even more higher resolutions are needed when we are interested in very detailed studies of chromospheric activity such as the analysis of the difference features present in the chromospheric emission line profiles, the study of chromospherically active binaries aimed to determine from which component of the binary belong the emission lines (see Montes et al. 1997b), or the analysis of the time variations and line asymmetries that occur during a stellar flare (see Montes et al. 1998b).

On the other hand, the simultaneous observations of different lines, that are formed at different height in the chromosphere (from the region of temperature minimum to the higher chromosphere), are of special interest for stellar activity studies since they provide very useful information about this stellar region. Ideally, simultaneous 
observations should be performed at all wavelengths in order to develop a coherent 3-D atmosphere model (see the multiwavelength optical observations of chromospherically active binary systems by Montes et al. 1997b, 1998a). So, to carry out these purposes applying the spectral subtraction technique, to as many lines as possible, a spectral library with a good spectral resolution and a good spectral coverage is needed.

The spectral library that we present in this paper is an extension of our previous one (Paper I) to higher spectral resolution covering a large spectral range. The library consist of echelle spectra of a sample of F, G, K and $\mathrm{M}$ field dwarf stars covering the spectral range from $4800 \AA$ to $10600 \AA$ and with spectral resolution ranging from 0.19 to $0.09 \AA$. These spectra include some of the spectral lines most widely used as optical and nearinfrared indicators of chromospheric activity such as: Na I $\mathrm{D}_{1}, \mathrm{D}_{2}$, and $\mathrm{Mg}$ I $\mathrm{b}$ triplet (formed in the upper photosphere and lower chromosphere), Ca II IRT lines (lower chromosphere), $\mathrm{H} \alpha, \mathrm{H} \beta$ (middle chromosphere), and He I $\mathrm{D}_{3}$ (upper chromosphere), as well as a large number of photospheric lines which can also be affected by chromospheric activity. Furthermore, the spectra also include a lot of lines of interest to spectral classification and calibration of temperatures purposes, as well as other lines normally used for the application of the Doppler imaging technique.

In Sect. 2 we report the details of our observations and data reduction. The library is presented in Sect. 3 with comments on the behaviour of some interesting spectral lines.

\section{Observations and data reduction}

The high-resolution echelle spectra presented here were obtained by us during several observing runs with the $4.2 \mathrm{~m}$ William Herschel Telescope (WHT) at La Palma Observatory, using the Utrecht Echelle Spectrograph (UES) mounted on a Nasmith focal station. A description of the WHT/UES is given by Unger (1992). In addition, we analysed also different WHT/UES observational campaigns retrieved from La Palma Data Archive (Zuiderwijk et al. 1994).

In Table 1 we give a summary of WHT/UES observations, for each observing run we list the date, the observer, the CCD detector, the number of echelle orders included, the central wavelength $\left(\lambda_{\mathrm{c}}\right)$, the wavelength range covered $\left(\lambda_{\mathrm{i}}-\lambda_{\mathrm{f}}\right)$ and the range of reciprocal dispersion achieved ( $\AA /$ pixel) from the first to the last echelle orders. The echelle grating used in all these runs was the E31. The interorder spacing was too small to perform a good sky subtraction. Sky contamination is only significant for the few faint very-late M-type stars of this library (VB 8 and VB 10). As can be seen in Table 1, the spectra cover the spectral range from $4800 \AA$ to $10600 \AA$ with spectral resolution ( $\mathrm{FWHM}=2$ pixels) ranging from 0.19 to $0.09 \AA$, corresponding to $R \sim 55000$. However, as the CCD chips we have used were smaller than the echelle orders, we did not get a full recording of the different orders. The gaps between the adjacent recorded orders got larger towards longer wavelengths, as can be seen in Tables 2 to 5 , where we give for each observing run the wavelength range and the spectral lines of interest in each echelle order.

The spectra have been extracted using the standard reduction procedures in the $\mathrm{IRAF}^{1}$ package (bias subtraction, flat-field division, and optimal extraction of the spectra). The wavelength calibration was obtained by taking spectra of a Th-Ar. Finally, the spectra have been normalized by a polynomial fit to the observed continuum.

\section{The library}

As in Paper I, the stars included in the library have been selected as stars with low levels of chromospheric activity, that is to say, stars that do not present any evidence of emission in the core of $\mathrm{Ca}$ II $\mathrm{H} \& \mathrm{~K}$ lines in our spectra (Montes et al. 1995c, 1996a), stars with the lower Ca II H \& K spectrophometric index S (Baliunas et al. 1995), or stars known to be inactive and slowly rotating stars from other sources (see Strassmeier et al. 1990; Strassmeier \& Fekel 1990; Hall \& Ramsey 1992). In addition, we provide spectra of some active stars of late and very-late spectral types.

Table 6 presents information about the observed stars. In this table we give the HD, HR and GJ numbers, name, spectral type and luminosity class $\left(T_{\mathrm{sp}}\right)$, from the Bright Star Catalogue (Hoffleit \& Jaschek 1982; Hoffleit \& Warren 1991) and the Catalogue of Nearby Stars (Gliese \& Jahreiss 1991), except for some M dwarfs for which we list the more recent spectral type determination given by Henry et al. (1994) and Kirkpatrick et al. (1995). In Col. (6) MK indicates if the star is included in the list of Morgan and Keenan (MK) Standard Stars compiled by García (1989). Column (7) give the metallicity [Fe/H] from Taylor $(1994 ; 1995)$ or Cayrel de Strobel $(1992 ; 1997)$ and Col. (8) rotational period $\left(P_{\text {rot }}\right)$ and $v \sin i$ from Donahue (1993); Baliunas et al. (1995); Strassmeier \& Fekel (1990); Fekel (1997); Stauffer \& Hartmann (1986); Martín et al. (1996), and Basri \& Marcy (1995; 1996). We also give, in Col. (9), the Ca II H \& $\mathrm{K}$ spectrophometric index S from Baliunas et al. (1995) and Duncan et al. (1991). In Col. (10) we list information about the observing run in which each star have been observed, using a code given in the first column of Table 1, and the last column indicate if the star was also included in Paper I.

In Fig. 1 we have plotted for a K1V star representative spectral orders, with the line identification marked. The two first orders $(\mathrm{H} \beta$ and $\mathrm{Mg}$ I b lines) correspond to the

1 IRAF is distributed by the National Optical Observatory, which is operated by the Association of Universities for Research in Astronomy, Inc., under contract with the National Science Foundation. 
K1V star HD 10476 from the Dec-93 observing run and the following orders correspond to K1V star HD 9546 from the the Nov.-94 run.

Representative spectra (from $\mathrm{F}$ to $\mathrm{M}$ stars) in different spectral regions are plotted in Figs. 2 to 6 in order to show the behaviour of the more remarkable spectroscopic features with the spectral type. In order of increasing wavelength we have plotted the following line regions: $\mathrm{H} \beta$ (Fig. 2), Mg I b triplet (Fig. 3) $\mathrm{Na}$ I $\mathrm{D}_{1}, \mathrm{D}_{2}$ (Fig. 4) and $\mathrm{He} \mathrm{I} \mathrm{D}_{3}$ ) (Fig. 4), $\mathrm{H} \alpha$ (Fig. 5), and Ca II IRT $\lambda 8498$, 8542, 8662 (Fig. 6).

In the following, we describe the behaviour of some interesting spectral lines and molecular bands present in the spectral range covered by the spectra (from 4800 to $10600 \AA$ ). We list the spectroscopic features in order of increasing wavelength, and the echelle order in which they appear in each observing run can be found in Tables 2 to 5 .

- The $\mathrm{H} \beta \lambda 4861.3$ line (Fig. 2) is a well know chromospherically activity indicator (emission or filled-in).

- The Mg I b triplet $\lambda \lambda 5167,5172,5183$ (Fig. 3) is luminosity sensitive in the range G8-K5. These strong neutral metal lines are formed in the lower chromosphere and the region of temperature minimum and they are good diagnostics of activity (Basri et al. 1989; Gunn \& Doyle 1997; Gunn et al. 1997; Montes et al. 1998b).

- The He I $\mathrm{D}_{3} \lambda 5876$ absorption line is another important indicator of stellar activity in the upper chromosphere (García López et al. 1993; Montes et al. 1997b, 1998a; Saar et al. 1997) and also could be in emission during stellar flares (Huenemoerder \& Ramsey 1987; Montes et al. 1996b, 1997b, 1998b).

- The Na I $D_{1} \lambda 5895.92$ and $D_{2} \lambda 5889.95$ lines (Fig. 4) are well known temperature and luminosity discriminant. These resonance lines are collisionally-controlled in the atmospheres of late-type stars and then provide information about chromospheric activity see Montes et al. (1996b, 1997b, 1998a) and the recent models of these lines for M dwarfs stars by Andretta et al. (1997).

- The wings of the Ca I triplet $\lambda \lambda 6102,6122,6162$ lines can be used as luminosity indicators (Cayrel et al. 1996). These lines are very weak at spectral type $\mathrm{F}$ and increase in strength with decreasing temperature.

- The V I $\lambda 6251.83$ and Fe I $\lambda 6252.57$ line-depth ratio can be used to determine stellar temperatures (Gray \& Johanson 1991; Gray 1994).

- The line ratio Fe II $\lambda 6432.65 / \mathrm{Fe}$ I $\lambda 6430.85$ is useful for spectral-class/temperature classification for $\mathrm{F}$ to $\mathrm{M}$ stars. Other spectral class indicators are the ratios of V I $\lambda 6452 / \mathrm{Ca}$ I $\lambda 6456$ (for $\mathrm{F}$, and $\mathrm{G}$ stars), Co I $\lambda 6455 / \mathrm{Ca}$ I $\lambda 6456$ and Fe II $\lambda 6457 / \mathrm{Ca}$ I $\lambda 6456$ (for F to K stars) (Strassmeier \& Fekel 1990).

- The Fe I $6411.66 \AA$, Fe I $6430.85 \AA$, and Ca I $6439.08 \AA$ lines normally used for the application of the Doppler imaging technique.
- The emission or filled-in of the $\mathrm{H} \alpha$ (6562.8 $\AA$ ) (Fig. 5) line is one the primary optical indicators of chromospheric activity in late-type stars, together with the Ca II $\mathrm{H} \& \mathrm{~K}$ emission lines.

- The Li I resonance line at $\lambda 6707.8 \AA$ and the subordinate lines at $\lambda 6103 \AA$ and $\lambda 8126 \AA$ (Pavlenko et al. 1995; Carlsson et al. 1994).

- The K I $\lambda 7664.91,7698.98$ doublet is a resonance transition and thus will be influenced by chromospheric activity, particularly in M dwarf stars (see Basri \& Marcy 1995; Schweitzer et al. 1996).

- The Rb I resonance line at $7947.63 \AA$ is strong in very late-type stars, and it seems to be a good temperature diagnostic (Basri \& Marcy 1995).

- The Na I doublet $\lambda \lambda 8183.26,8194.8$ lines are subordinate transitions and therefore, form mainly in the photosphere and should not be significantly affected by the chromosphere. These lines can be used as a dwarf/giant indicator (Schiavon et al. 1997).

- The Ti I lines for multiplet 33 (specially the $\lambda 8382.54$, 8382.82 lines) and other Ti I lines. can be used as a luminosity classification criterium because they present a positive luminosity effect (Keenan \& Hynek 1945; Ginestet et al. 1994; Jashek \& Jashek 1995; Montes et al. 1998a).

- The Ca II infrared triplet (IRT) $\lambda \lambda 8498,8542$, and 8662 lines (Fig. 6) formed in the lower chromosphere and are also important activity indicators (Linsky et al. 1979; Foing et al. 1989; Dempsey et al. 1993; Montes et al. 1998a). These lines have been also used by several authors as a gravity sensitive index. In this

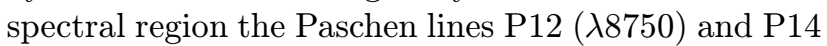
$(\lambda 8598)$ are also visible in $\mathrm{F}$ stars (Carquillat et al. 1997).

- From mid K through $M$ stars we can also see a number of titanium oxide ( $\mathrm{TiO})$ molecular bands such as (5847-6058), (6080-6390), (6322-6512), (6569-6649), (6651-6852), (7053-7270), (76667861), (8206-8569), (8432-8452), and (8859-8937), useful in classifying early $\mathrm{M}$ dwarfs, and a number of vanadium oxide (VO) bands such as (7400-7510), (7851-7973), and (8521-8668), useful in classifying late $\mathrm{M}$ dwarfs. $\mathrm{CaH}$ bands at $(6346,6382,6389)$ and (6750-7050) are also present in these stars.

- Other strong features that appear in the spectra are the telluric $\mathrm{O}_{2} \mathrm{~A}$ and $\mathrm{B}$ bands at $6867 \AA 7600 \AA$ and several telluric $\mathrm{H}_{2} \mathrm{O}$ bands at (7186-7273), (8164-8177), 8227, 8282, (8952-8972), and (8980-8992).

A more detailed description of the $\mathrm{H} \alpha, \mathrm{H} \beta, \mathrm{Na}$ I $\mathrm{D}_{1}$, $\mathrm{D}_{2}$ and $\mathrm{He} \mathrm{I}_{3}$ lines and the corresponding photospheric features included in these spectral regions, can be found in Paper I. An extensive list of features identifiable in late$\mathrm{K}$ to late-M spectra from 6300 to $9000 \AA$ can be found in Kirkpatrick et al. (1991). A description of the spectroscopic characteristics of very cool dwarfs and substellar 
candidates is given by Martín et al. (1996). For more information about spectral classification of stars and the behaviour of chemical elements in stars the reader is referred to Jaschek \& Jaschek (1990; 1995).

In order to enable other investigators to make use of the spectra of this library, all the multidimensional spectra containing all the echelle orders of the stars listed in Table 6 are available as FITS format files at the CDS in Strasbourg, France, via anonymous ftp to cdsarc.ustrasbg.fr (130.79.128.5). They are also available via the World Wide Web at:

http://www.ucm.es/OTROS/Astrof/fgkmsl/UESfgkmsl.html.

In order to facilitate the use of this library the onedimensional normalized and wavelength-shifted spectra, resulting for the extraction of the orders containing the more remarkable spectroscopic features (the spectral regions plotted in Figs. 2 to 6), are also available as separate FITS format files.

The extension of this library including stars of higher luminosity class, as well as the use of these spectra to analyse temperature sensitive lines in order to improve the actual line-depth ratio temperature calibrations (Gray \& Johanson 1991; Gray 1994) and spectralclass/temperature classifications (Strassmeier \& Fekel 1990), will be the subject of forthcoming papers.

Table 1. Summary of WHT/UES observations (published only electronically at CDS)

Table 2. UES spectral orders (Jul.-93) (published only electronically at CDS)

Table 3. UES spectral orders (Dec.-93) (published only electronically at CDS)

Table 4. UES spectral orders (Apr.-94, Mar.-95, Jun.-95) (published only electronically at CDS)

Table 5. UES spectral orders (Nov.-94) (published only electronically at CDS)
Acknowledgements. We thank J. Sanz-Forcada for help in the reduction of some of the echelle spectra. This research has made use the La Palma Archive and of the SIMBAD data base, operated at CDS, Strasbourg, France. This work has been supported by the Universidad Complutense de Madrid and the Spanish Dirección General de Investigación Científica y Técnica (DGICYT) under grants PB94-0263 and PB95-1132C02-01.

\section{References}

Andretta V., Doyle J.G., Byrne P.B., 1997, A\&A 322, 266

Baliunas S.L., Donahue R.A., Soon W.H., et al., 1995, ApJ 438, 269

Basri G., Wilcots E., Stout N., 1989, PASP 101

Basri G., Marcy G.W., 1995, AJ 109, 762

Basri G., Marcy G.W., 1996, in Cool Stars, Stellar Systems, and the Sun, Ninth Cambridge Workshop, Pallavicini R. and Duppree A.K. (eds.), ASP Conf. Ser. 109, 587

Byrne P.B., 1993, A\&A 272, 495

Carquillat J.M., Jashek C., Jashek M., Ginestet N., 1997, A\&AS 123,5

Carlsson M., Rutten R.J., Bruls J.H.M.J., Shchukina N.G., 1994, A\&A 288, 860

Cayrel de Strobel G., Hauck B., Francois P., et al., 1992, A\&AS 95, 273

Cayrel de Strobel G., Soubiran C., Friel E.D., Ralite N., Francois, P., 1997, A\&AS 124, 299

Cayrel R., Faurobert-Scholl M., Feautrier N., Spielfieldel A., Thevenin F., 1996, A\&A 312, 549

Dempsey R.C., Bopp B.W., Henry G.W., Hall D.S., 1993, ApJS 86, 293

Donahue R.A., 1993, Ph.D. Thesis, New Mexico State University

Duquennoy A., Mayor M., 1991, A\&A 248, 485

Fekel F.C., 1997, PASP 109, 514

Foing B., Crivellari L., Vladilo G., Rebolo R., Beckman J., 1989, A\&AS 80, 189

García B., 1989, Bull. Inform. CDS 36, 27

García López R.J., Rebolo R., Beckman J.E., McKeith C.D., 1993, A\&A 273, 482

Gliese W., Jahreiss H., 1991, Preliminary Version of the Third Catalogue of Nearby Stars. Astron. Rechen-Institut, Heidelberg

Ginestet N., Carquillat J.M., Jashek M., Jashek C., 1994, A\&AS 108, 359

Gray D.F., Johanson H.L., 1991, PASP 103, 439

Gray D.F., 1994, PASP 106, 1248

Gunn A.G., Doyle J.G., 1997, A\&A 318, 60

Gunn A.G., Doyle J.G., Houdebine E.R., 1997, A\&A 319, 211

Hall J.C., Ramsey L.W., 1992, AJ 104, 1942

Henry, T.J., Kirkpatrick, J.D., Simons, D.A., 1994, AJ 108, 1437

Hoffleit D., Jaschek C., 1982, The Bright Star Catalogue, 4th ed. New Haven: Yale University Press

Hoffleit D., Warren W. 1991, The Bright Star Catalogue, 5th ed., in Astronomical Data Center CD ROM, Selected Astronomical Catalogs Vol. 1, Brotzman L.E., Gessmer S.E (eds.)

Huenemoerder D.P., Ramsey L.W., 1987, ApJ 319, 392

Keenan P.C., Hynek J.A., 1945, ApJ 101, 265 
Fig. 1. Representative spectral orders for the Dec.-93 and Nov.-94 observing runs of a K1V star, with the line identification marked (published only electronically at CDS)

Table 6. Stars

\begin{tabular}{|c|c|c|c|c|c|c|c|c|c|c|c|}
\hline HD & $\mathrm{HR}$ & GJ & Name & $T_{\mathrm{sp}}$ & MK & $\begin{array}{l}{[\mathrm{Fe} / \mathrm{H}]} \\
(\mathrm{dex})\end{array}$ & $\begin{array}{l}P_{\text {rot }} \\
\text { (days) }\end{array}$ & $\begin{array}{l}v \sin i \\
\left(\mathrm{~km} \mathrm{~s}^{-1}\right)\end{array}$ & $S$ & Obs. & Pap. I \\
\hline \multicolumn{12}{|l|}{ F stars } \\
\hline 33256 & 1673 & 189.2 & 68 Eri & $\mathrm{F} 2 \mathrm{~V}$ & & -0.49 & - & 0 & - & 3 & \\
\hline 6406 & & & & F3/F5IV/V & & - & - & - & - & 3 & \\
\hline 84937 & & & $\mathrm{BD}+142151$ & F5VI & & -1.86 & - & - & - & 2 & \\
\hline & & & $\mathrm{BD}+183423$ & $\mathrm{~F} 6 \mathrm{~V}$ & & - & - & - & - & 1 & \\
\hline 78154 & 3616 & & $\sigma^{2} \mathrm{UMa}$ & F6IV & & - & - & 0 & 0.142 & 6 & \\
\hline 9826 & 458 & 61 & 50 And & F8V & MK & -0.14 & - & 8 & 0.154 & 1 & \\
\hline 142373 & 5914 & 602 & $\chi \mathrm{Her}$ & $\mathrm{F} 8 \mathrm{~V}$ & & -0.431 & - & 10.0 & 0.147 & 5 & $*$ \\
\hline 144284 & 5986 & 609.1 & $\theta$ Dra & F8IV (SB1) & & 0.23 & - & 27.7 & 0.203 & 6 & \\
\hline 98230 & 4374 & $423 \mathrm{~B}$ & $\xi \mathrm{UMa} \mathrm{B}$ & $\mathrm{F} 8.5 \mathrm{~V}$ & MK & -0.12 & - & 3 & - & 5 & \\
\hline 114762 & & & $\mathrm{BD}+182700$ & F9V & & -0.87 & - & - & - & 3 & \\
\hline 79028 & 3648 & 337.1 & $16 \mathrm{UMa}$ & F9V (SB1) & & - & - & 0 & - & 5,6 & \\
\hline 114710 & 4983 & 502 & $\beta$ Com & F9.5V & MK & 0.135 & 12.35 & $4.3 \mathrm{~F}$ & 0.201 & 3 & $*$ \\
\hline \multicolumn{12}{|l|}{ G stars } \\
\hline 160269 & 6573 & & 26 Dra & G0IV-V (MK) (SB1) & MK & - & - & 41 & - & 1 & \\
\hline 15335 & 720 & 99.1 & 13 Tri & G0V & & - & - & $<6$ & - & 4 & \\
\hline 39587 & 2047 & $222 \mathrm{~B}$ & $\chi^{1}$ Ori & G0V (SB1) & MK & -0.084 & 5.36 & $8.6 \mathrm{~F}$ & 0.325 & 3 & $*$ \\
\hline $98231 \mathrm{~A}$ & 4375 & $423 \mathrm{~A}$ & $\hat{\xi} \mathrm{UMa} A$ & G0V (SB1) & & -0.352 & - & $<15$ & - & 5 & $*$ \\
\hline 84737 & 3881 & & $15 \mathrm{LMi}$ & G0.5V & MK & -0.04 & - & 3 & 0.145 & 5 & \\
\hline 10307 & 483 & 67 & $\mathrm{BD}+41328$ & G1.5V (SB1) & MK & 0.14 & - & 2.1 & 0.152 & 2 & \\
\hline 42807 & 2208 & 230 & $\mathrm{BD}+101050$ & $\mathrm{G} 2 \mathrm{~V}$ & & - & - & - & 0.352 & 4 & \\
\hline 153631 & & 650 & BD-13 4528 & G2V (SB1) & & - & - & - & - & 3 & \\
\hline 186427 & 7504 & $765.1 \mathrm{~B}$ & 16 Cyg B & G3V & $\mathrm{MK}$ & -0.002 & - & 0.4 & 0.145 & 1 & $*$ \\
\hline 86728 & 3951 & 376 & $20 \mathrm{LMi}$ & G3V & $\mathrm{MK}$ & -0.11 & - & 3 & 0.156 & 6 & \\
\hline 115617 & 5019 & 506 & $61 \mathrm{Vir}$ & G5V & & 0.032 & - & 0.4 & 0.162 & 3 & $*$ \\
\hline 178428 & 7260 & 746 & $\mathrm{BD}+163752$ & G5V (SB1) & & - & - & - & 0.154 & 3,6 & \\
\hline 33802 B & & & BD-12 1095B & G5Ve & & - & - & - & - & 3 & \\
\hline 149414 & & $629.2 \mathrm{~A}$ & BD-03 3968 & G5V (SB1) & & -1.14 & - & - & - & $3,5,6$ & \\
\hline 20630 & 996 & 137 & $\kappa^{1}$ Cet & G5V & MK & 0.133 & 9.24 & 3.9 & 0.366 & 2 & $*$ \\
\hline 31966 & & 182.1 & $\mathrm{BD}+14804$ & G5V & & - & - & - & - & 4 & \\
\hline 108754 & & 469.1 & BD-02 3528 & G7V (SB1) & & - & - & - & - & 3,5 & \\
\hline $131156 \mathrm{~A}$ & $5544 \mathrm{~A}$ & $566 \mathrm{~A}$ & $\xi$ Boo A & G8V & MK & -0.151 & 6.31 & 3.2 & 0.461 & 5 & * \\
\hline 44867 & 2302 & & $\mathrm{BD}+161135$ & G8IV (G9III) & & - & - & - & - & 4 & \\
\hline 195987 & & 793.1 & $\mathrm{BD}+413799$ & G9V (SB1) & & - & - & - & - & 3 & \\
\hline \multicolumn{12}{|l|}{ K stars } \\
\hline 10780 & 511 & 75 & $\mathrm{BD}+63238$ & $\mathrm{KOV}$ & & 0.36 & - & 0.6 & 0.280 & 4 & \\
\hline 185144 & 7462 & 764 & $\sigma$ Dra & K0V & MK & -0.045 & - & 0.6 & 0.215 & 1 & $*$ \\
\hline 18972 & & & $\mathrm{BD}+13494$ & KOIV & & - & - & - & - & 4 & \\
\hline 48432 & 2477 & & 13 Lyn & KOIII & & - & - & $<19$ & 0.120 & 2 & \\
\hline 9546 & & 59.3 & ADS $1233 \mathrm{~A}$ & $\mathrm{~K} 1 \mathrm{~V}$ & & - & - & - & - & 4 & \\
\hline 10476 & 493 & 68 & 107 Psc & K1V & MK & -0.123 & 35.2 & 0. & 0.198 & 2 & $*$ \\
\hline 76291 & 3545 & & $\mathrm{BD}+461459$ & K1IV & & - & - & - & - & 2 & \\
\hline 6027 & & 50.1 & $\mathrm{BD}+58155$ & K2V (K3III) & & - & - & - & - & 4 & \\
\hline 101177 B & $4486 \mathrm{~B}$ & $433.2 \mathrm{~B}$ & ADS $8250 \mathrm{~A}$ & K2V (SB) & & - & - & - & 0.144 & 3,6 & \\
\hline 38392 & 1982 & $216 \mathrm{~B}$ & $\gamma$ Lep B & $\mathrm{K} 2 \mathrm{~V}$ & & 0.02 & - & - & - & 3 & \\
\hline 136713 & & 1191 & BD-10 4088 & $\mathrm{~K} 2 \mathrm{~V}$ & & - & - & - & - & 3 & \\
\hline 223778 & 9038 & $909 \mathrm{~A}$ & $\mathrm{BD}+741047$ & K3V & & - & - & - & - & 1 & \\
\hline 219134 & 8832 & 892 & $\mathrm{BD}+562966$ & K3V & MK & -9.000 & - & - & 0.230 & 2 & $*$ \\
\hline $16160 \mathrm{~A}$ & 753 & $105 \mathrm{~A}$ & $\mathrm{BD}+06398$ & $\mathrm{~K} 3 \mathrm{~V}$ & MK & -0.297 & 48.0 & - & 0.226 & 4 & $*$ \\
\hline 98800 & & $2084 \mathrm{~A}$ & $\operatorname{ADS} 8141 \mathrm{~A}$ & $\mathrm{~K} 4 \mathrm{~V}$ & & - & - & - & - & 3,5 & \\
\hline $131156 \mathrm{~B}$ & $5544 \mathrm{~B}$ & $566 \mathrm{~B}$ & $\xi$ Boo B & $\mathrm{K} 4 \mathrm{~V}$ & & 0.19 & 12.28 & 20 & 1.381 & 5 & $*$ \\
\hline 12208 & & 83.3 & V598 Cas & $\mathrm{K} 5 \mathrm{~V}$ & & - & - & - & - & 4 & \\
\hline 154363 & & 653 & BD-04 4225 & K5V & & - & - & - & - & 3 & \\
\hline 201091 & 8085 & $820 \mathrm{~A}$ & 61 Cyg A & $\mathrm{K} 5 \mathrm{~V}$ & MK & -0.06 & 35.37 & 0.6 & 0.658 & 2,6 & $*$ \\
\hline \multirow[t]{2}{*}{201092} & 8086 & $820 \mathrm{~B}$ & 61 Cyg B & $\mathrm{K} 7 \mathrm{~V}$ & MK & -0.10 & 37.84 & 1.4 & 0.986 & 2,6 & $*$ \\
\hline & & 52 & $\mathrm{BD}+63137$ & $\mathrm{~K} 7 \mathrm{~V}$ & & - & - & - & - & 4 & \\
\hline 157881 & & 673 & $\mathrm{BD}+023312$ & $\mathrm{~K} 7 \mathrm{~V}$ & & 0.40 & - & 3.9 & 1.464 & 1 & \\
\hline 88230 & & 380 & $\mathrm{BD}+501725$ & $\mathrm{~K} 7 \mathrm{~V}(1)(\mathrm{K} 6 \mathrm{~V} \mathrm{MK})$ & MK & 0.28 & - & 3.1 & 1.617 & 3 & \\
\hline 151877 & & 639 & $\mathrm{BD}+372804$ & $\mathrm{~K} 7 \mathrm{~V}$ & & - & - & - & 0.197 & 3 & \\
\hline 151288 & & 638 & $\mathrm{BD}+332777$ & $\mathrm{~K} 7.5 \mathrm{Ve}$ & MK & - & - & - & 1.380 & 3,6 & \\
\hline
\end{tabular}


Table 6. continued

\begin{tabular}{|c|c|c|c|c|c|c|c|c|c|c|c|}
\hline HD & $\mathrm{HR}$ & GJ & Name & $T_{\mathrm{sp}}$ & MK & $\begin{array}{l}{[\mathrm{Fe} / \mathrm{H}]} \\
(\mathrm{dex})\end{array}$ & $\begin{array}{l}P_{\text {rot }} \\
\text { (days) }\end{array}$ & $\begin{array}{l}v \sin i \\
\left(\mathrm{~km} \mathrm{~s}^{-1}\right)\end{array}$ & $S$ & Obs. & Pap. I \\
\hline \multicolumn{12}{|l|}{ M stars } \\
\hline & & 16 & & MoV & & - & - & - & - & 1 & \\
\hline \multirow[t]{2}{*}{79210} & & $338 \mathrm{~A}$ & ADS $7251 \mathrm{~A}$ & MoVe (1) & & - & - & - & 2.113 & 3 & $*$ \\
\hline & & 572 & $\mathrm{BD}+452247$ & MOV & & - & - & - & - & 3,5 & \\
\hline 232979 & & 172 & $\mathrm{BD}+52857$ & $\mathrm{M} 0.5 \mathrm{~V}$ & MK & - & - & - & 1.909 & 4 & \\
\hline $1326 \mathrm{~A}$ & & $15 \mathrm{~A}$ & GX And & M1.5V (1) (M2V MK) & MK & - & - & - & - & 4 & \\
\hline 36395 & & 205 & BD-03 1123 & M1.5V (1) & MK & 0.60 & - & - & - & 4 & \\
\hline \multirow[t]{3}{*}{95735} & & 411 & $\mathrm{BD}+362147$ & $\mathrm{M} 2 \mathrm{~V}$ & MK & -0.20 & - & - & 0.424 & 3 & \\
\hline & & $623 \mathrm{AB}$ & LHS 417 & M2.5V (1) & & - & - & - & - & $3,5,6$ & \\
\hline & & 813 & LHS 3605 & M3V & & - & - & - & - & 1 & \\
\hline $173739 \mathrm{~A}$ & & $725 \mathrm{~A}$ & ADS $11632 \mathrm{~A}$ & M3V (1) & & - & - & - & 0.534 & 3,5 & \\
\hline \multirow[t]{2}{*}{180617} & & $752 \mathrm{~A}$ & LHS 473 & M3 V (1) & MK & - & - & - & 1.252 & 6 & \\
\hline & & 273 & $\mathrm{BD}+051668$ & M3.5V (1) & & - & - & - & - & 3 & \\
\hline \multirow[t]{2}{*}{$16160 \mathrm{~B}$} & & $105 \mathrm{~B}$ & $\mathrm{BD}+06398 \mathrm{~B}$ & M3.5V (1) (3) & & - & - & - & - & 1 & \\
\hline & & 699 & Barnard's star & M4V (1) & & - & - & - & - & 3 & \\
\hline \multirow[t]{2}{*}{13124} & & 748 & Wolf 1062 & M4V (M3.5V (4)) & & - & - & - & - & $3,5,6$ & \\
\hline & & 447 & FI Vir, LHS 315 & M4V (1) (3) & & - & - & - & - & 6 & \\
\hline \multirow[t]{10}{*}{12025} & & & U Per & M4III (M6e) & & - & - & - & - & 4 & \\
\hline & & $234 \mathrm{AB}$ & LHS $1849 / 50$ & $\mathrm{M} 4.5 \mathrm{~V}(1) \mathrm{e}$ & & - & - & $<10$ & - & 3,5 & \\
\hline & & $831 \mathrm{AB}$ & LHS 511 & $\mathrm{M} 4.5 \mathrm{~V}(1) \mathrm{e}$ & & - & - & $<10$ & - & 6 & \\
\hline & & $473 \mathrm{AB}$ & FL Vir, LHS 333 & $\mathrm{M} 5.5 \mathrm{~V}(1) \mathrm{e}$ & & - & - & - & - & $3,5,6$ & \\
\hline & & $1245 \mathrm{~A}$ & V1581 Cyg & $\mathrm{M} 5.5 \mathrm{~V} \mathrm{e}$ & & - & - & - & - & 6 & \\
\hline & & 1245B & LHS 3495 & $\mathrm{M} 5.5 \mathrm{~V} \mathrm{e}$ & & - & - & - & - & 6 & \\
\hline & & 406 & LHS 36 & M6V (1) e & & - & - & $<3$ & - & 3 & $*$ \\
\hline & & 1111 & DX Cnc, LHS 248 & M6.5V & & - & - & 11 & - & 3,5 & $*$ \\
\hline & & $644 \mathrm{C}$ & VB 8, LHS 429 & M7V (2) & & - & 0.14 & 8 & - & $3,5,6$ & \\
\hline & & $752 \mathrm{~B}$ & VB $10, \mathrm{~V} 1298 \mathrm{Aql}$ & M8V (1) e & & - & - & $<5$ & - & 6 & \\
\hline
\end{tabular}

(1): Henry et al. (1994)

(2): Kirkpatrick et al. (1995)

(3): "Zero H $\alpha$ star", Byrne (1993)

(4): Kirkpatrick et al. (1991)

MK: "A List of MK Standard Stars", García (1989)

SB: Spectroscopic Binary (Duquennoy \& Mayor 1991; Mazeh et al. 1997).

Kirkpatrick J.D., Henry T.J., McCarthy D.W. Jr., 1991, ApJS 77,417

Kirkpatrick, J.D., Henry, T.J., Simons, D.A., 1995, AJ 109, 797

Jaschek C., Jaschek M., 1990, "The Classification of Stars". Cambridge University Press

Jashek C., Jashek M., 1995, "The Behavior of chemical elements in stars". Cambridge University Press

Linsky J.L., Hunten D., Glacken D., Kelch W., 1979, ApJS 41, 481

Martín E.L., Rebolo R., Zapatero-Osorio M.R., 1996, ApJ 469, 706

Martín E.L., Zapatero-Osorio M.R., Rebolo R., 1996, in Cool Stars, Stellar Systems, and the Sun, Ninth Cambridge Workshop, R. Pallavicini and A.K. Duppree (eds.), ASP Conf. Ser. 109, 615

Mazeh T., Martín E.L., Goldberg D., Smith H.A., 1997, MNRAS 284, 341

Montes D., Fernández-Figueroa M.J., De Castro E., Cornide M., 1995a, A\&A 294, 165

Montes D., Fernández-Figueroa M.J., De Castro E., Cornide M., 1995b, A\&AS 109, 135

Montes D., De Castro E., Fernández-Figueroa M.J., Cornide M., 1995c, A\&AS 114, 287

Montes D., Fernández-Figueroa M.J., Cornide M., De Castro E., 1996a, A\&A 312, 221

Montes D., Sanz-Forcada J., Fernández-Figueroa M.J.,
Lorente R., 1996b, A\&A 310, L29

Montes D., Martín E.L., Fernández-Figueroa M.J., Cornide M., De Castro E., 1997a, A\&AS 123 (Paper I)

Montes D., Fernández-Figueroa M.J., De Castro E., Sanz-Forcada J., 1997b, A\&AS 125, 263

Montes D., Sanz-Forcada J., Fernández-Figueroa M.J., De Castro E., Poncet A., 1998a, A\&A 330, 155

Montes D., Saar S.H., Collier Cameron A., Unruh Y.C., 1998b, MNRAS (in preparation)

Pavlenko Y.V., Rebolo R., Martín E.L., García López R.J., 1995, A\&A 303, 807

Saar S.H., Huovelin J., Osten R.A., Shcherbakow A.G., 1997, A\&A 326, 741

Schiavon R.P., Barbury B., Rossi S.C.F., Milone A., 1997, ApJ 479, 902

Schweitzer A., Hauschildt P.H., Allard F., Basri G., 1996, MNRAS 283, 821

Stauffer J.R., Hartmann L.W., 1986, ApJS 61, 531

Strassmeier K.G., Fekel F.C., Bopp B.W., Dempsey R.C., Henry G.W., 1990, ApJS 72, 191

Strassmeier K.G., Fekel F.C., 1990, A\&A 230, 389

Taylor B.J., 1994, PASP 106, 704

Taylor B.J., 1995, PASP 107, 734

Unger S., 1992 UES Users' Manual, WHT, Roque de Los Muchachos Observatory

Zuiderwijk E.J., Martin R., Raimond E., van Diepem G.N.J., 1994, PASP 106, 515 


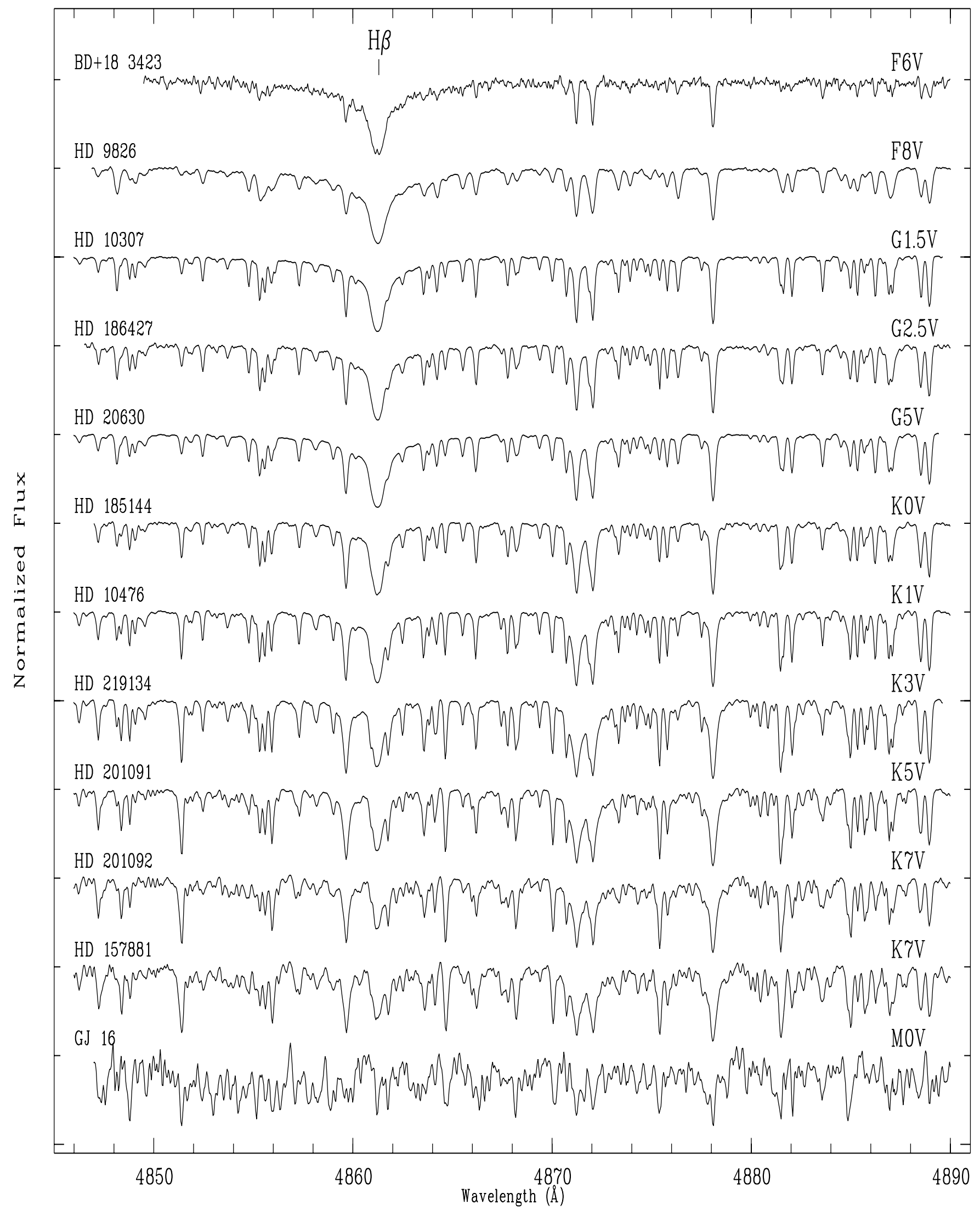

Fig. 2. Spectra in the $\mathrm{H} \beta$ line region 


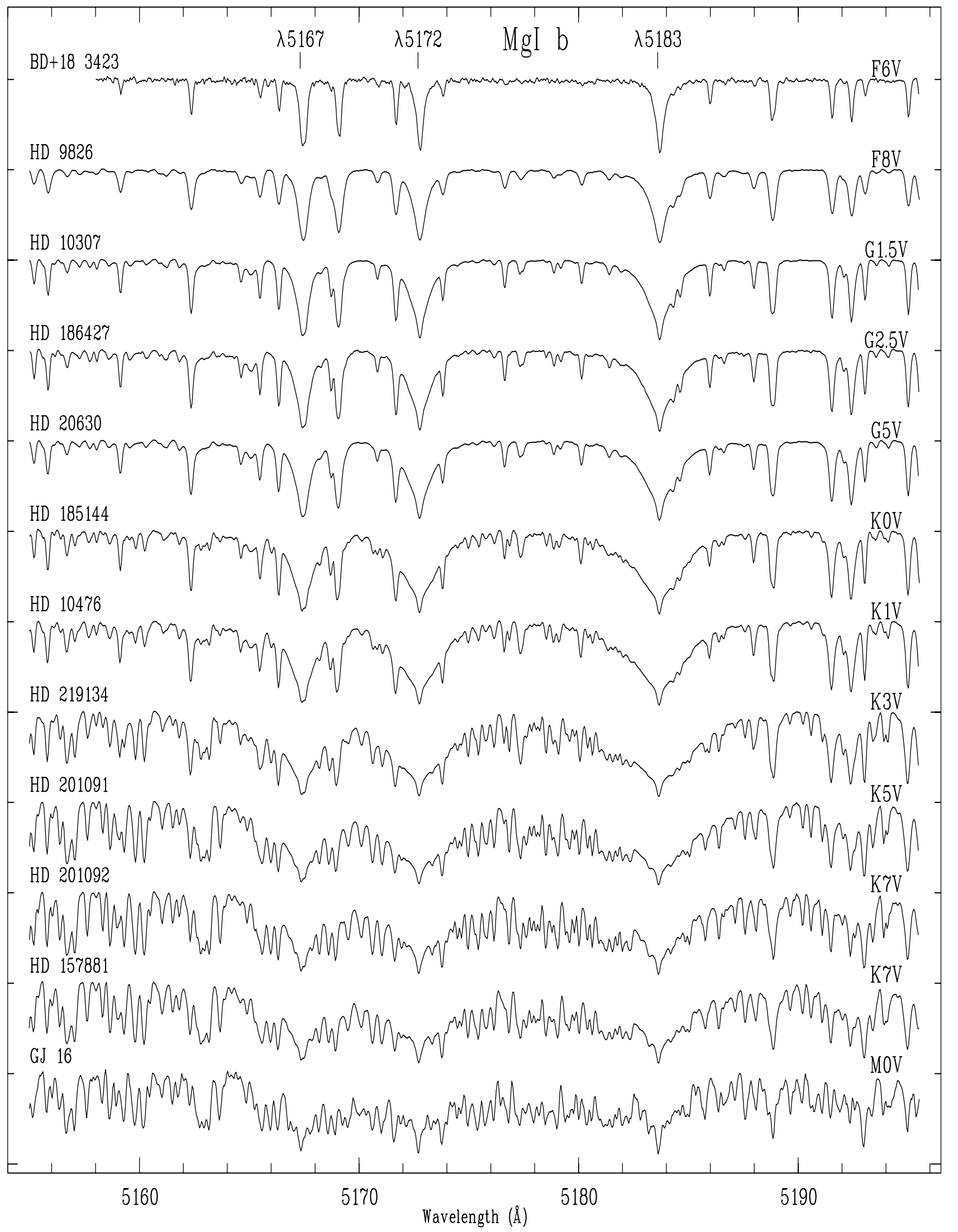

Fig. 3. Spectra in the $\mathrm{Mg}$ I b triplet lines region 


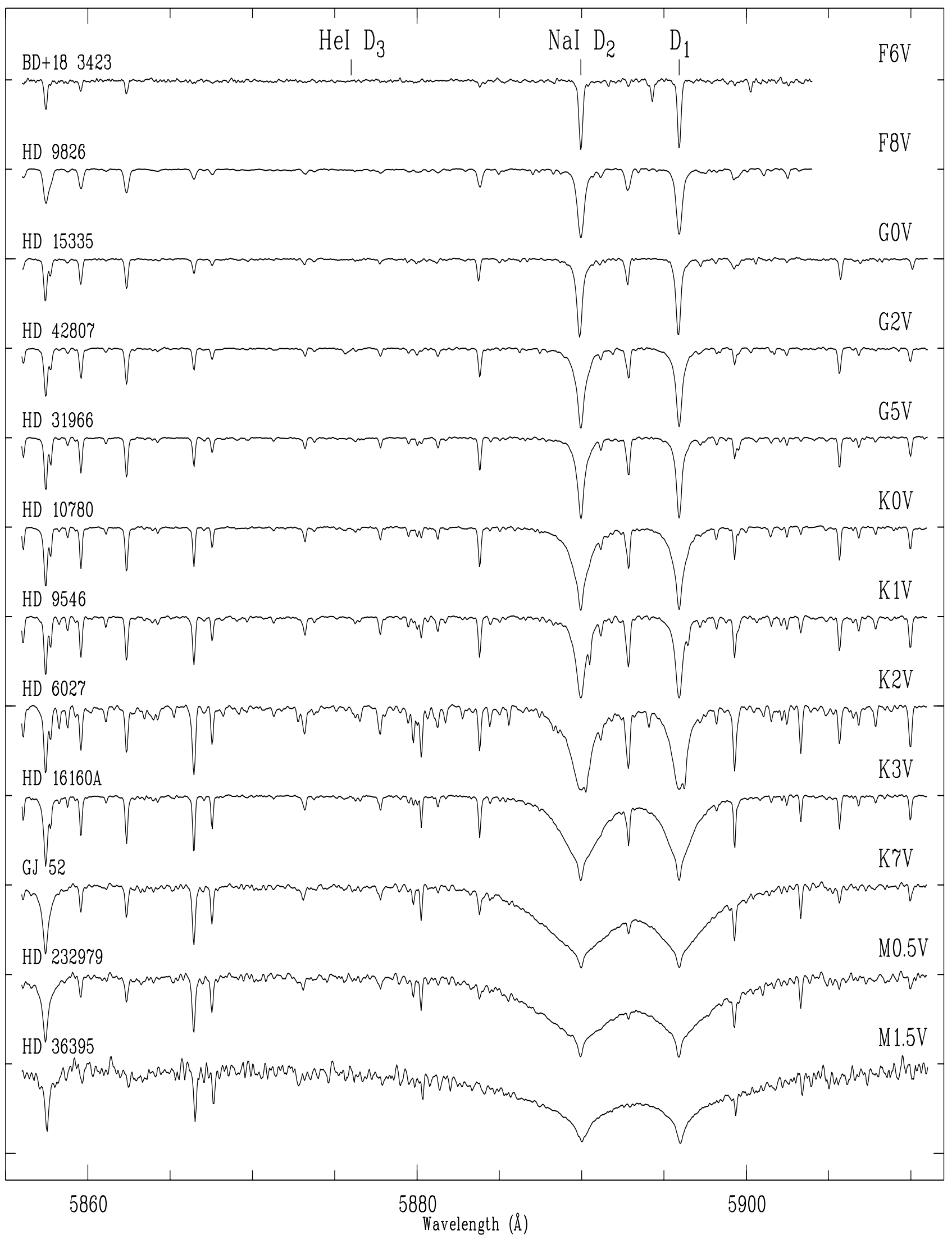

Fig. 4. Spectra in the $\mathrm{Na} \mathrm{I}_{1}, \mathrm{D}_{2}$ and $\mathrm{He} \mathrm{I}_{3}$ lines region 


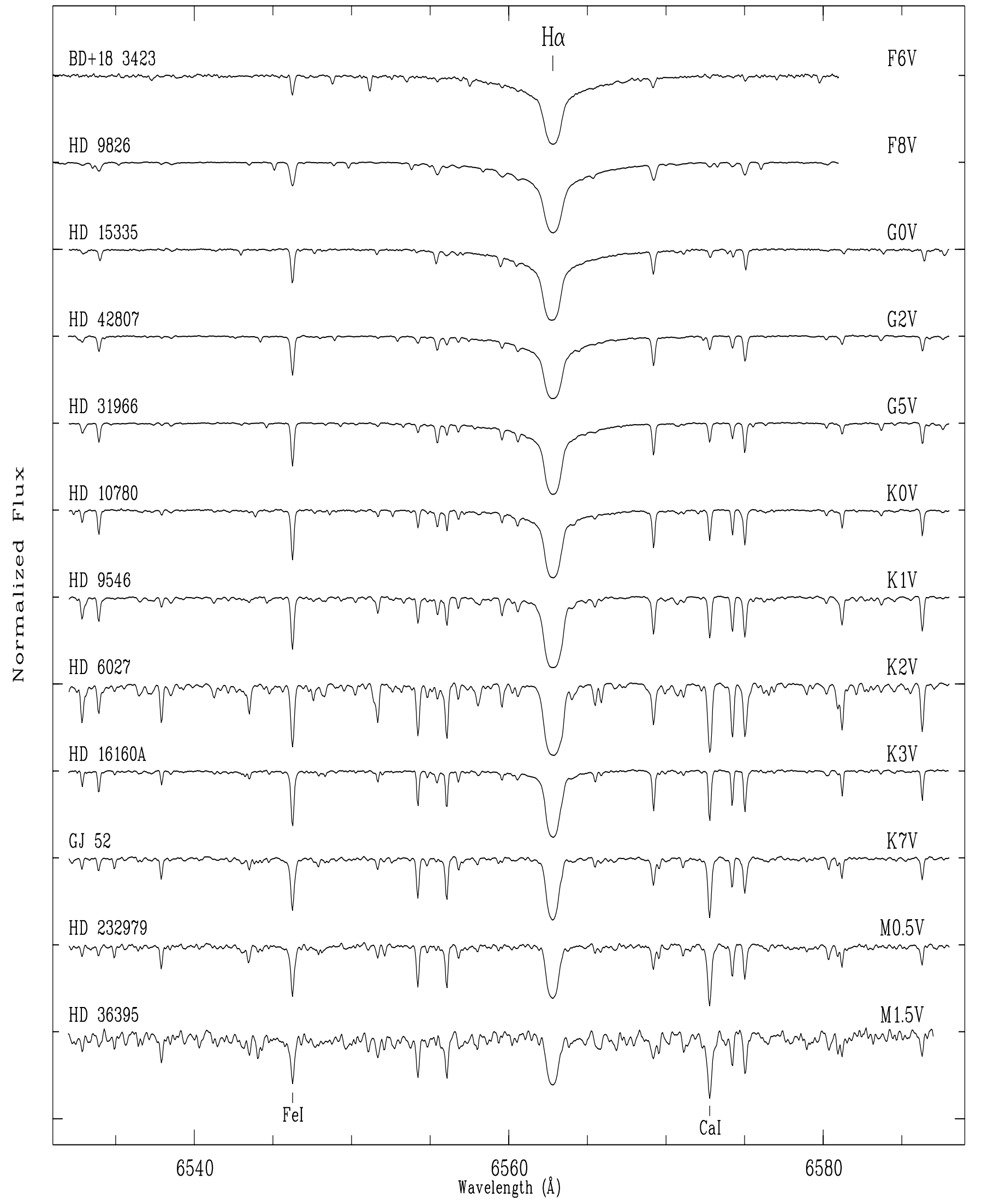

Fig. 5. Spectra in the $\mathrm{H} \alpha$ line region 


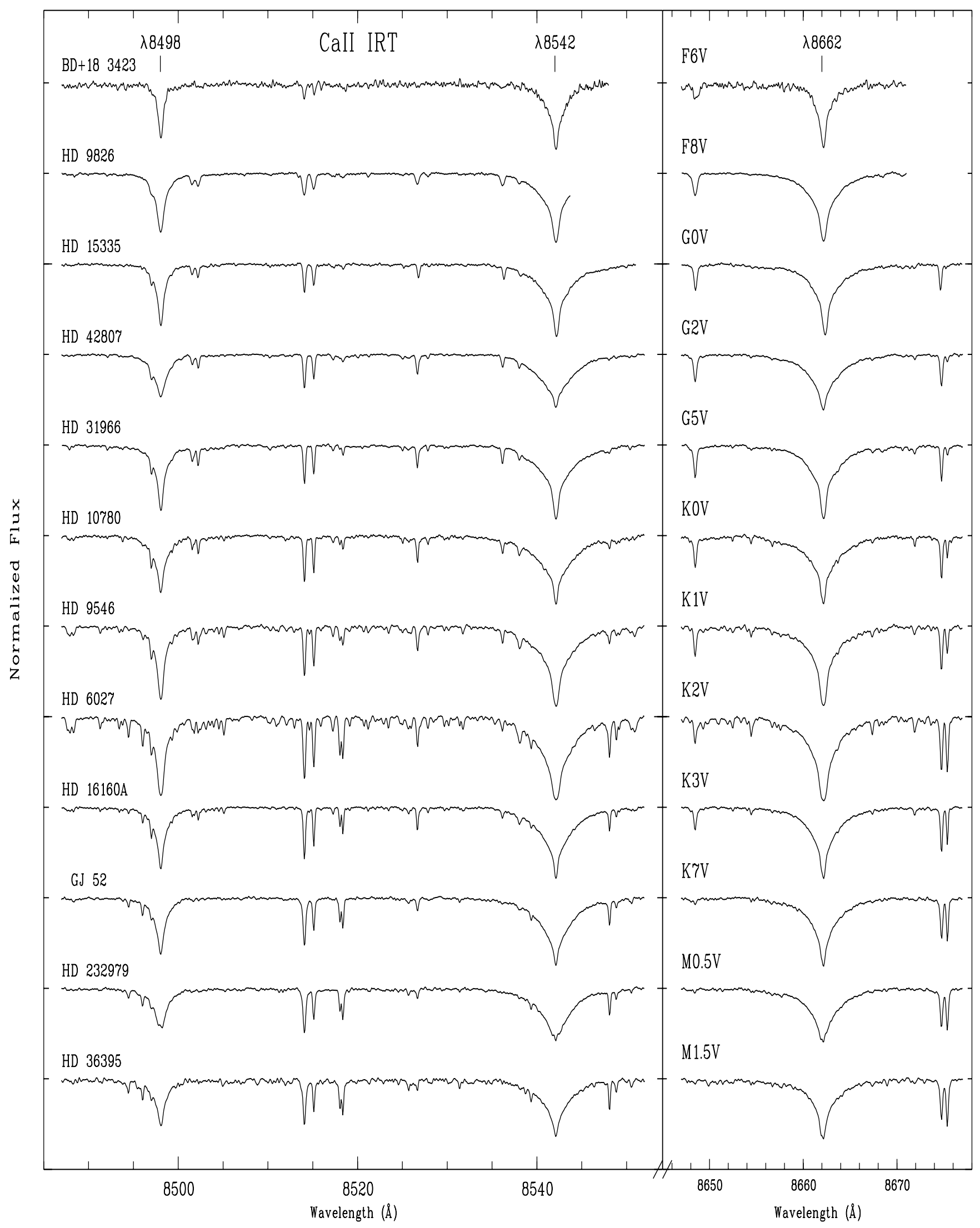

Fig. 6. Spectra in the Ca II IRT $\lambda 8498,8542,8662$ lines region 